\title{
The Impact on Children's Mental Health of School Closures to Prevent the Spread of the Novel Coronavirus (COVID-19)
}

\author{
Yuma Ishimoto $^{1 *}$, Takahiro Yamane ${ }^{2}$, Yuki Matsumoto ${ }^{3}$, and Katsutoshi Kobayashi ${ }^{4}$
}

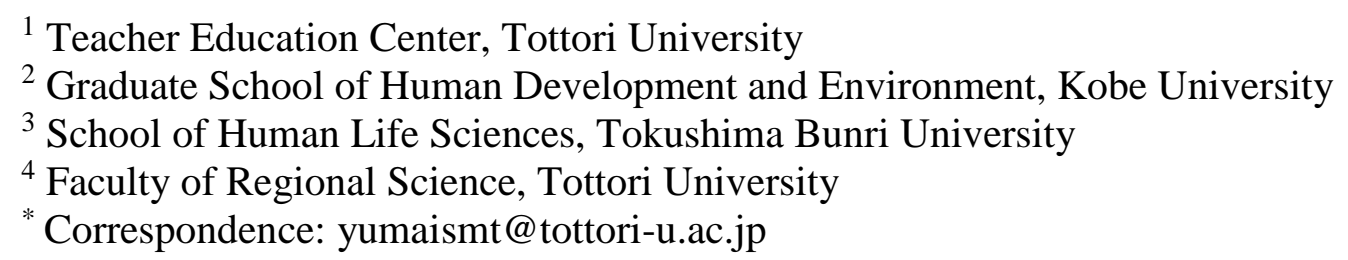

\begin{abstract}
In Japan, schools were suddenly closed all over the country with one month left at the end of the school year to prevent the spread of new coronavirus infections. Although previous studies have shown that quarantine and isolation have a negative impact on mental health, the impact of school closure on children's mental health is not clear. This study examined mental health indicators, such as emotional symptoms, in addition to behavior during school closure and anxiety after school resumes, among children in three primary schools for which survey data existed in December before school closure. No cases of infection have been confirmed in the area where the school was located at the time of the survey. As a result, anxiety about whether they would be able to return to their normal routine after school resumed was associated with mental health. In addition, there was a worsening of emotional symptoms in those who were in medium group of school adjustment as of December.
\end{abstract}

Keywords: Covid-19, School Closure, Elementary School, Emotional Symptoms, Japan

\section{Previous research}

\section{Introduction}

In order to prevent the spread of the novel coronavirus (COVID-19), schools have been closed around the world and many children have been affected. To date, there have been several studies of the psychological impacts of quarantine and social isolation during the spread of infectious diseases, which mostly have addressed issues of health care staff and a few of the general public. For instance, Hawryluck et al. (2004) surveyed 18-year-old and older subjects who underwent voluntary quarantine during the SARS epidemic and showed that the experience of quarantine increased PTSD symptoms, which became more pronounced the longer the quarantine period continued. Jeong et al. (2016) surveyed adult subjects who underwent quarantine during the MERS epidemic and found a specific number of individuals who experienced anxiety symptoms, anger, and other similar emotional problems during the quarantine. Since children show different reactions than from adults' ones during disasters, it is conceivable that children would show similarly specific responses during quarantine and social isolation as well; however, there is too 
little research targeting children so that it is not clear what type of psychological reactions they would show. The only available study targeting children surveyed parents or guardians about impacts on children and revealed that children who had experienced quarantine and social isolation fulfilled the criteria for PTSD at a similar ratio to other traumatic events such as disasters (Sprang \& Silman, 2013). Their research also uncovered diagnoses of stress disorder, adjustment disorder, grief, and the like, indicating that experiences of quarantine and social isolation harm children's psychological health. However, this research utilized responses of parents or guardians and did not directly question children, so its accuracy could arguably be insufficient. Also, Hawryluck et al. (2004) and Sprang \& Silman (2013) surveyed respondents who voluntarily took part in the study, so there is the possibility that said participants had a stronger desire to share their experiences if they were more personally affected by the infectious disease (Cava et al., 2005). Hence, there could be limits to generalizing the results of such surveys soliciting participants on a voluntary basis.

\section{Measures in Japan and their impact}

To prevent the spread of novel coronavirus infections in Japan, on Friday, February 28th, 2020, the Prime Minister directed the temporary closure of elementary, junior high, senior high, and special education schools. The closure affected on the following Monday, March 2nd, was supposed to continue through the end of the academic year and the beginning of spring break (around March 24th). While the directive did not have a legal foundation, data from March 4 showed compliance from $98.8 \%$ of public elementary schools, $99.0 \%$ of public junior high schools, and all national elementary schools, junior high schools (MEXT; Ministry of Education, Culture, Sports, Science and Technology, 2020a). In practical terms, the directive entailed more than a month-long closure, since the spring break until April 6th when a new academic year would start. In Japan, there are occasional temporary school closures for individual grades or entire schools in regions when hit by disasters (earthquakes, torrential rainfalls, etc.), or more commonly due to the spread of influenza infections. In 2009, when there was a concern over the spread of a novel influenza strain, the Ministry of Health, Labour and Welfare (MHLW) directed the temporary (approximately one week) closure of junior high schools and senior high schools in Osaka and Hyogo prefectures, as well as some elementary schools in the region. Thus, short-term school closure measures have been implemented in Japan; however, this is our first time to experience the nationwide month-long school closure.

With the current school closure measures at issue in Japan, the MEXT initially asked schools and municipalities to instruct children to stay inside generally (MEXT, 2020b), and some schools guided students not to go outside (Ozaki, 2020). There was a reiteration on March 9th, ten days after the request for school closure, stating that a reasonable level of outdoor exercise such as walking was encouraged (MEXT, 2020c). Despite this, as calls arose for nationwide self-restraint concerning events (MHLW, 2020a), multiple events targeting children were canceled, and it appeared that a significant number of children were forced to spend most of their time inside their homes. Japanese research (Mizumoto, Yamamoto, \& Nishiura, 2013) conducted during school closures during the spread of the novel influenza strain in 2009 similarly showed that $80 \%$ of children did not go outside their homes except in urgent situations. While the current school closure measures technically differ from quarantine, they are more likely to affect a de facto nearquarantine. 
Furthermore, before the sudden, long-lasting measures taken at the end of the academic term, schools and households were not given sufficient time to prepare for school closure adequately. Researchers have shown that confusion has ensued in case that children and their parents or guardians learned of school closures in the media before the school report (Braunack-Mayer et al., 2013). In the current situation, the announcement was suddenly made public by the Prime Minister through the press, so the confusion can be estimated to be proportionally large. Additionally, due to little time to mentally prepare, the measures presumably would lead to a tremendous psychological impact on related people. A survey of parents or guardians with children affected by the current school closures (NPO Florence, 2020) showed that $56.8 \%$ of the respondents agreed with an item stating that they were worried about their children's "stress and mental health due to the closures and due to not being able to see their friends." In short, they were concerned about psychological influences on their children. Besides, academic concerns might associate with anxiety. Compared to China, where the infection originated, and immediate measures for school closure were taken with strategies of providing online learning and materials (Wang et al., 2020), Japanese schools mostly had no such plans in evidence. As the school closure continued, the issue of significant anxiety over children's school academic activity accumulates.

\section{Purpose of the study}

The present research presented here sought to clarify what type of psychological costs damaged children by this sudden, protracted school closure. Psychological impacts of school closures on children would differ significantly according to the presence or absence of alternative measures, the number of infectious patients in proximity, the existence of "stay at home" orders, and so on. By accumulating knowledge of psychological impacts in each set of circumstances, we may be able to obtain a more detailed understanding of these effects on children who must stay home when school is closed. This paper's research project is one undertaking within this more significant inquiry. Even during the time of increasing infection, we should not underestimate the question of whether psychological costs may surpass the epidemiological benefit of forced, large-scale quarantines (Rubin \& Wessely, 2020). Our project aimed to form an understanding of the psychological costs, which in the future would contribute to considering strategies to mitigate said costs when faced with a similar predicament preemptively. Additionally, we might be able to compare the psychological prices and the effects of infectious disease prevention and then consider (or reconsider) measures similar to closing schools.

This research surveyed a cohort of schoolchildren from schools in a December 2019 school survey with a different purpose addressing school adaptation and psychological adaptation. A research approach that surveys students at only one point in time during or after school closure cannot accurately assess whether the survey answers reveal the impact of school closure. In order to allow for accurate assessment, it is necessary to use a longitudinal approach starting before school closure and continuing during school closure. Participants who completed the Strengths and Difficulties Questionnaire (SDQ; Goodman, 1997) in December 2019 received a second SDQ during this period, which allowed for assessing changes in emotional problems. Although the SDQ seldom surveys of stress reactions during emergencies such as disasters, it can help us to understand 
emotional reactions (anxiety, fear, and the like), as well as the difficulty of maintaining attention span. Answers from students themselves, not from parents or guardians, could be useful for more accurate understandings of psychological impacts on students' wellbeing.

This research also surveyed student conduct during school closure, and with whom they frequently spent their time. In addition, the objective of including the school data in this research schools that had been surveyed in December with regard to school adaptation and emotional problems the previous year was also to shed light on the type of impact that pre-closure psychological risks had on psychological adaptation during the school closure. Previous research during school closures (Braunack-Mayer et al., 2013) has shown that students acted according to rules based on individual, unique criteria. The finding clarified that some students never left their homes, while others behaved in such a way that they did not differ from their usual schedules (other than going to school). It has also been indicated that children's communication with parents is essential for maintaining psychological adjustment (Wang et al., 2020); however, in Japan, it is not very easy for employees to take paid leave, meaning that many workers cannot take time off from work, so there is concern that some children do not have sufficient communication with their parents or guardians. In actual surveys of parents and guardians, $25 \%$ of parents and guardians of 1st to 3rd-grade elementary school students and approximately half of parents and guardians of 4th grade or older elementary school students and junior and senior high school students reported that they left their child/ren at home alone for extended periods (NPO Florence, 2020). Additionally, in surveys of respondents who experienced social isolation during the MERS epidemic in South Korea, it has been shown that a history of mental illness was a risk factor for anxiety symptoms (Jeong et al., 2016). It is conceivable that the degree of school adaptation and emotional problems at the time of the pre-closure survey had an impact on emotional problems during school closure.

\section{Method}

\section{Procedures}

Participants

Participants were children from three elementary schools (A, B, and C) for which survey data existed as of December. Table 1 shows target school years and the like. All of these schools are located in one provincial prefecture where no cases of COVID-19 have been confirmed at the time of the survey, but more than 100 cases have been confirmed in neighboring prefecture. All of the schools were closed from February 29 and, as of the time of the survey, none of the children were going to school.

Table1 School profile

\begin{tabular}{llrlrr}
\hline & Type & Grade & Method & \multicolumn{1}{c}{$n$} & $\%$ \\
\hline A & national & 3 to 6 & mail back & 165 & 63.2 \\
B & municipal & 3 to 5 & mail back & 55 & 35.3 \\
C & municipal & 3 to 6 & FTF & 75 & - \\
\hline
\end{tabular}




\section{Survey Period}

The survey was conducted in late March.

\section{Survey Procedure}

At subject school A, the questionnaire was distributed to parents or guardians during a 3day period when they were instructed to come and retrieve items from school. Participants were asked to return the questionnaires by mail. At subject schools $\mathrm{B}$, the questionnaire was distributed at an end-of-term ceremony, which was the first day of visiting campus after the school closure. Participants were asked to return the questionnaires by mail. At subject school $\mathrm{C}$, the questionnaire was distributed and collected by home room teachers when they visited students at home.

\section{Ethical Considerations}

Given that this survey was intended to serve as reference and support material provided by the school after the end of the school closure, participation was, in principle, requested from all students. The questionnaire clearly stated in writing that the responses were in no way related to academic grades and that students would incur no disadvantage from the nature of their responses. In addition, materials were distributed to parents stating that they would be excluded from the research data if they did not want their responses to be used for research purposes. None of the parents refused to have their children's responses used for research purposes. The study protocol was reviewed and approved by the Institutional Review Boards of the institution which first author belongs to.

\section{Structure of the Questionnaire}

Scale Related to Questions on Conduct and Emotions

This survey used the Japanese version of the self-report SDQ (for students age 11 to 17) created by Goodman (1997) and available on the SDQ website. The SDQ is composed of five subscales: "conduct problems," "hyperactivity/inattention," "emotional symptoms," "peer relationship problems," and "prosocial behavior," and the scores of the four subscales except "prosocial behavior" are added together to generate a total difficulty score. The reliability and validity of the Japanese version have been verified by Noda et al. (2012). For each question item, respondents are asked to answer on a 3-point scale from "not true" ( 0 points) to "certainly true" ( 2 points). The process of response analysis utilizes the subtotals of each subscale and the total difficulties score (the subtotal of four of the subscales).

\section{Anxiety Over the Current Situation and the Future}

Three questions were asked covering: anxiety over the infection, anxiety over changes in life (whether they will be able to return to their usual life after the school closure), and anxiety over falling behind scholastically. Responses were solicited on a five-point scale ranging from 1 (does not apply) to 5 (applies).

Who children spent time with during the school closure

Students were asked who they spent most of their time with during the closure. Answer options included: alone, with mother, father, grandmother, grandfather, sibling(s), other family member(s), friend(s), after-school day care instructor/extracurricular lesson instructor, and other. Respondents were asked to give numerical answers $(1,2,3)$ ranking options from most time to third most time. 
How time was spent during the school closure

Questions expected to relate to mental and physical adaptation asked about how time was spent during the closure. Elementary school students received 13 questions. The specific items were shown in Table3. Responses were solicited on a four-point scale ranging from 1 (not at all), 2 (not much: once or twice a week), 3 (often: three or four times a week), to 4 (almost daily).

\section{Cover Sheet}

The cover sheet of the questionnaire asked for the subject's full name, grade, class (within their grade), and gender. Students at schools whose responses were collected by mail were also asked to give the date that they filled in their responses.

\section{Pre-Survey}

As mentioned above, an earlier survey (initially for a different purpose) had been conducted in December. In this preliminary survey, all questionnaires were distributed in class by homeroom teachers. Participants were given time to fill in the forms and submit them soon after.

\section{Structure of the Questionnaire Form of Pre-Survey}

Scale of Emotional Problems

Of the five subscales in the Japanese version of the SDQ for 11 to 17 year olds, only five items related to emotional symptoms were used. For each question item, respondents are asked to answer on a 3-point scale from "not true" ( 0 points) to "certainly true" ( 2 points).

\section{Scale Relating to School Adaptation}

The School Adaptation Scale by Furuichi and Tamaki (1994) was used to measure school adaptation. The reliability and validity of the School Adaptation Scale (comprising 10 items) have been verified by Furuichi and Tamaki (1994). For each item, respondents were asked to answer on a 5-point scale from "does not apply" (1 point) to "applies" (5 points). Analysis utilized the averages of scores from the 10 items.

\section{Cover Sheet}

The cover sheet of the questionnaire asked for the subject's full name, grade, class (within their grade), and gender.

\section{Outline of the Participants}

\section{Result}

The number of people in the analysis by grade is shown in Table 2 . The response rates for the target schools that collected the questionnaires by mail are shown in Table 1 . The average response date for the targeted schools that collected the questionnaires by mail was 27.4 days ( $S D=4.3$ ), with February 29, the first day of the school year when the school closures effectively began. 
Table2 Number of students by grade

\begin{tabular}{llllrr}
\hline & 3 & 4 & 5 & \multicolumn{1}{r}{6} & \\
\hline A & 44 & 50 & 41 & 30 & 165 \\
B & 19 & 22 & 14 & 0 & 55 \\
C & 17 & 20 & 18 & 20 & 75 \\
\hline
\end{tabular}

\section{How to spend their time during school closure}

Table 3 shows the average values for each item by grade level for how they spend their time during school closure. Contacting friends via the Internet tended to increase as grades progressed, but other behaviors showed little difference between grades. Although the school was closed, many of them went out of the house, perhaps because there were no confirmed cases of infection in the area.

Table3 Grade level averages for behavior during school closure

\begin{tabular}{|c|c|c|c|c|c|c|c|c|c|}
\hline & & \multicolumn{2}{|c|}{3} & \multicolumn{2}{|c|}{4} & \multicolumn{2}{|c|}{5} & \multicolumn{2}{|c|}{6} \\
\hline & & $M$ & $S D$ & $M$ & $S D$ & $M$ & $S D$ & $M$ & $S D$ \\
\hline 1 & I spent time going to extracurricular lessons. & 1.9 & .9 & 2.0 & 1.0 & 2.0 & 1.0 & 2.0 & .8 \\
\hline 2 & I spent time playing with friends. & 1.8 & .9 & 1.8 & 1.0 & 1.6 & .7 & 1.8 & .8 \\
\hline 3 & I spent time outside the house. & 3.0 & .9 & 3.1 & .8 & 3.0 & .9 & 2.9 & 1.0 \\
\hline 4 & I spent time doing schoolwork. & 3.5 & .8 & 3.6 & .7 & 3.6 & .8 & 3.4 & .7 \\
\hline 5 & I woke up late in the morning. & 2.6 & 1.0 & 2.6 & 1.0 & 2.6 & 1.0 & 2.7 & 1.1 \\
\hline 6 & I stayed up late at night. & 2.5 & 1.0 & 2.5 & 1.0 & 2.7 & 1.1 & 2.4 & 1.1 \\
\hline 7 & I ate my meals alone. & 1.2 & 6 & 1.3 & 6 & 1.4 & .9 & 1.5 & .9 \\
\hline 8 & I went out with my family member(s). & 2.3 & .7 & 2.4 & .9 & 2.2 & .7 & 2.1 & .7 \\
\hline 9 & $\begin{array}{l}\text { I played video games on my smartphone, mobile game system, } \\
\text { or in-home game system for more than an hour. }\end{array}$ & 2.3 & 1.2 & 2.3 & 1.2 & 2.9 & 1.2 & 2.6 & 1.2 \\
\hline 10 & I contacted my friend(s) using my smartphone or PC. & 1.1 & .2 & 1.2 & .5 & 1.4 & .9 & 2.0 & 1.3 \\
\hline 11 & I watched the news on the novel coronavirus on $\mathrm{TV}$, on my smartphone, etc. & 3.1 & 1.1 & 3.3 & 1.0 & 3.4 & .9 & 3.5 & .8 \\
\hline 12 & I was physically active, such as taking walks or exercising. & 2.7 & 1.0 & 3.0 & .8 & 3.0 & 1.0 & 2.8 & 1.0 \\
\hline 13 & I went to an after-school daycare center. & 1.4 & .8 & 1.6 & 1.1 & 1.1 & .5 & 1.2 & .6 \\
\hline
\end{tabular}

\section{Who they spent their time with during school closure}

In response to the question about who they spent most of their time with during school closure, those selected as those who spent the most are shown in Table 4 by grade level. There was not much difference by grade level, as many spent most of their time with their sibling(s) or their mother.

\section{Association of behavioral/emotional problems with anxiety about the current situation and future and behavior while school closure}

In order to examine the association of behavioral/emotional problems with anxiety about 
Table4 The person they spent most of their time with during the school closure (\%)

\begin{tabular}{llrrrr}
\hline & & 3 & \multicolumn{1}{c}{5} & \multicolumn{1}{c}{6} \\
\hline 1 & alone & 7.5 & 1.1 & 11.0 & 8.0 \\
2 & mother & 15.0 & 29.3 & 15.1 & 10.0 \\
3 & father & 1.3 & 6.5 & 6.8 & .0 \\
4 & grandmother & 10.0 & 10.9 & 9.6 & 2.0 \\
5 & grandfather & .0 & 3.3 & 4.1 & .0 \\
6 & sibling(s) & 32.5 & 22.8 & 32.9 & 34.0 \\
7 & other family member(s) & .0 & 3.3 & 1.4 & .0 \\
8 & friend(s) & 3.8 & 2.2 & .0 & 4.0 \\
9 & after-school day care instructor/extracurricular lesson instructor & 6.3 & 9.8 & 1.4 & .0 \\
10 & other & 2.5 & 1.1 & .0 & .0 \\
\hline
\end{tabular}

note. The total does not add up to $100 \%$ because some perticipant listed more than one.

the current situation and the future and behavior during school closure, their correlation coefficients were calculated (Table 5). As a result, in terms of anxiety about the current situation and the future, anxiety about whether they will be able to return to their usual life was moderately positively correlated with the total difficulties score, and was also weakly positively associated with emotion symptoms, conduct problems, and hyperactivity/inattention. Anxiety about infection and falling behind in studies were also positively associated with total difficulty scores and emotional symptom, but they were relatively low. In terms of behavior while school closure, staying up late showed a weak positive association with total difficulty score, conduct problems, and hyperactivity/inattention, and studying showed a weak negative association with conduct problems and hyperactivity/inattention. Other behaviors were rarely associated with SDQ.

Table5 Association of behavioral/emotional problems with anxiety about the current situation and future and behavior while school closure

\begin{tabular}{|c|c|c|c|c|c|c|c|c|c|c|c|c|}
\hline & \multicolumn{2}{|c|}{ TDS } & \multicolumn{2}{|c|}{ ES } & \multicolumn{2}{|c|}{$\mathrm{CP}$} & \multicolumn{2}{|c|}{ HI } & \multicolumn{2}{|c|}{ PP } & \multicolumn{2}{|c|}{ PB } \\
\hline & $r$ & $p$ & $r$ & $p$ & $r$ & $p$ & $r$ & $p$ & $r$ & $p$ & $r$ & $p$ \\
\hline a anxiety over the infection & .25 & .000 & .32 & .000 & .08 & .158 & .11 & .061 & .08 & .186 & -.04 & .549 \\
\hline b anxiety over changes in life & .41 & .000 & .39 & .000 & .25 & .000 & .23 & .000 & .16 & .024 & -.13 & .053 \\
\hline c anxiety over falling behind scholastically & .25 & .000 & .25 & .000 & .11 & .059 & .16 & .005 & .13 & .032 & -.03 & 641 \\
\hline 1 going to extracurricular lessons & -.12 & .044 & -.02 & .773 & -.12 & .043 & -.19 & .001 & -.03 & .639 & .11 & .052 \\
\hline 2 playing with friends & -.02 & .766 & .00 & .961 & -.02 & .805 & -.03 & .615 & -.05 & .417 & .05 & .367 \\
\hline 3 going outside & -.03 & .628 & -.04 & .476 & .02 & .760 & .02 & .777 & -.07 & .220 & -.05 & .398 \\
\hline 4 studying & -.19 & .001 & -.08 & .194 & -.21 & .000 & $\underline{-.23}$ & .000 & -.02 & .739 & .17 & .004 \\
\hline 5 waking up late & .12 & .043 & .03 & .593 & .14 & .022 & .14 & .016 & .01 & .888 & -.06 & .333 \\
\hline 6 staying up late at night & .32 & .000 & .20 & .001 & .32 & .000 & .25 & .000 & .08 & .197 & -.15 & .013 \\
\hline 7 eating my meals alone & .09 & .141 & .10 & .084 & .03 & .678 & .04 & .487 & .05 & .388 & -.01 & .899 \\
\hline 8 going out with my family & .00 & .977 & .08 & .159 & -.03 & .609 & -.08 & .172 & -.02 & .782 & .16 & .006 \\
\hline 9 playing games for more than an hour & .17 & .005 & .12 & .049 & .18 & .002 & .15 & .009 & -.03 & .638 & -.03 & .671 \\
\hline 10 contact my friend(s) over smartphone or PC & .03 & .584 & .02 & .732 & .05 & .366 & .02 & .772 & -.07 & .243 & -.03 & 627 \\
\hline 11 watching the news on the novel coronavirus & -.15 & .015 & -.07 & .228 & -.05 & .430 & -.16 & .006 & -.14 & .017 & .05 & .413 \\
\hline 12 being physically active & -.20 & .004 & -.19 & .004 & -.10 & .164 & -.12 & .079 & -.10 & .168 & .03 & 612 \\
\hline 13 going to an after-school daycare center & .00 & .984 & -.01 & .843 & .01 & .937 & -.01 & .858 & .03 & .712 & .13 & .062 \\
\hline
\end{tabular}




\section{The relationship between spending time alone and behavioral/emotional problems}

In order to examine the effects of whether or not they spent much time alone during school closure on behavioral/emotional problems, the total difficulties score and each subscale score were compared between those who answered between 1 and 3 about "alone" and those who did not in response to the question of who they spent time with during school closure. The results showed no significant differences in the total difficulties score or in any of the subscales.

\section{Comparison and correlation between the emotional symptoms scores in December and during the school closure}

An overall and grade-wise paired t-test was conducted to identify changes in scores on emotional symptoms at December and during the school closure. As a result, there was no significant change in the overall results or in any of the grades (Table 6). To examine the stability of scores on emotional symptom, the correlation coefficients between scores on emotional symptoms as of December and scores during school closure were calculated by grade level. The results showed that the correlation tended to increase as the grade level increased, indicating that the stability of the scores increased (Table 6).

Table6 Comparison and correlation of emotional symptoms.

\begin{tabular}{|c|c|c|c|c|c|c|c|c|c|c|}
\hline \multirow{2}{*}{ grade } & \multirow{2}{*}{$n$} & \multicolumn{2}{|c|}{ December } & \multicolumn{2}{|c|}{ Closure } & \multirow[b]{2}{*}{$l$} & \multirow{2}{*}{$p$} & \multirow{2}{*}{$g_{\text {adj }}$} & \multirow{2}{*}{$r$} & \multirow{2}{*}{$p$} \\
\hline & & $\bar{M}$ & $S D$ & $M$ & $\overline{S D}$ & & & & & \\
\hline whole & 280 & 2.7 & 2.6 & 2.8 & 2.3 & -1.1 & .289 & .06 & .48 & .000 \\
\hline 3 & 74 & 3.3 & 2.8 & 3.5 & 2.6 & -.7 & .510 & .09 & .38 & .001 \\
\hline 4 & 88 & 2.5 & 2.6 & 2.3 & 1.9 & .9 & .363 & .11 & .41 & .000 \\
\hline 5 & 69 & 2.5 & 2.5 & 2.9 & 2.3 & -1.6 & .124 & .17 & .57 & .000 \\
\hline 6 & 49 & 2.2 & 2.3 & 2.6 & 2.4 & -1.5 & .148 & .18 & .62 & .000 \\
\hline
\end{tabular}

\section{The Impact of School Adjustment on Emotional Symptoms at December.}

An analysis with a latent change model was conducted to examine the impact of school adjustment at December on changes in emotional symptoms from December to the time of school closure. Latent change models can decouple the initial value (intercept) and change (slope) of a variable to examine the change between two time points and the influence of other variables on that change (McArdle \& Nesselroade, 1994; Takahashi, 2017). Controlling for grade and gender, we examined whether school adjustment as of December had an effect on changes in emotional symptoms (Table 7). The overall goodness of fit of the model was good (CFI=.963, RMSEA=.038). Both intercept and slope variances were significant $(\mathrm{p}<.001)$. School adjustment scores at December indicated a significant negative path to initial values of emotional symptoms. The correlation coefficient between initial values and change was -.61, a moderate negative correlation, indicating that those with higher school adjustment at December had lower initial values of emotional symptoms and those with lower initial values of emotional symptoms had greater change in emotional symptoms. 
Table7 Results of the latent change model

\begin{tabular}{|c|c|c|c|c|c|c|}
\hline & \multicolumn{3}{|c|}{ Intercept } & \multicolumn{3}{|c|}{ Slope } \\
\hline & $B$ & $\beta$ & $p$ & $B$ & $\beta$ & $p$ \\
\hline Grade & -.06 & -.16 & .014 & .02 & .08 & .287 \\
\hline Gender & -.10 & -.13 & .043 & .07 & .11 & .135 \\
\hline School Adaptation & -.09 & -.22 & .001 & .01 & .03 & .697 \\
\hline
\end{tabular}

\section{Differences in Changes in Emotional Symptoms depending on School Adjustment Scores in December}

School adjustment in December did not significantly affect the slope of emotional symptoms, so school adjustment did not directly affect the amount of change in emotional symptoms. However, there was a significant negative effect from school adjustment on the initial value of emotional symptoms, and a significant negative correlation between the initial value of emotional symptoms and the amount of change, suggesting that school adjustment affects the amount of change in emotional symptoms via the initial value of emotional symptoms. Therefore, in order to explore how changes in emotional symptoms differ according to school adjustment in December, we divided the children into three groups (high, meddle, and low) with approximately the same number of children based on the average $\pm 0.5 \mathrm{SD}$ of school adjustment in December. Paired t-tests were conducted for each group to examine changes in emotional symptoms (Table 8). The results showed a significant difference only in the middle group, with a worsening of emotional symptoms.

Table8 Differences in Changes in Emotional Symptoms depending on School Adjustment Scores at December

\begin{tabular}{|c|c|c|c|c|c|c|c|c|}
\hline & & \multicolumn{2}{|c|}{ December } & \multicolumn{2}{|c|}{ Closure } & & \multirow{2}{*}{$p$} & \multirow{2}{*}{$g_{\text {adj }}$} \\
\hline & & $M$ & $S D$ & $M$ & $S D$ & & & \\
\hline high & 97 & 2.0 & 2.5 & 2.0 & 2.0 & .1 & .900 & .00 \\
\hline middle & 87 & 2.6 & 2.6 & 3.2 & 2.5 & -2.0 & .049 & .20 \\
\hline low & 87 & 3.2 & 2.5 & 3.2 & 2.3 & .0 & .969 & .01 \\
\hline
\end{tabular}

\section{Differences in behavior during school closure by school adjustment as of December}

An analysis of variance was conducted in order to analyze the differences in the behavior of each group during school closure (Table 9), since the worsening of emotional symptoms was found only in the middle group in the analysis conducted on the basis of the school adjustment value at December. The results showed that there was a difference between the groups in terms of extracurricular lessons, schoolwork, staying up late, and games. Multiple comparisons showed significant differences between the low and medium groups for extracurricular lessons, between the low and medium groups, and between the low and high groups for schoolwork and games, and between the low and high groups for staying up late. The extracurricular lessons was done more by the middle group than the low group. The schoolwork was done more by the middle and high groups than the low group. The staying up late was done more by the low group than high group. The game was more of a low group than a medium or high group. 


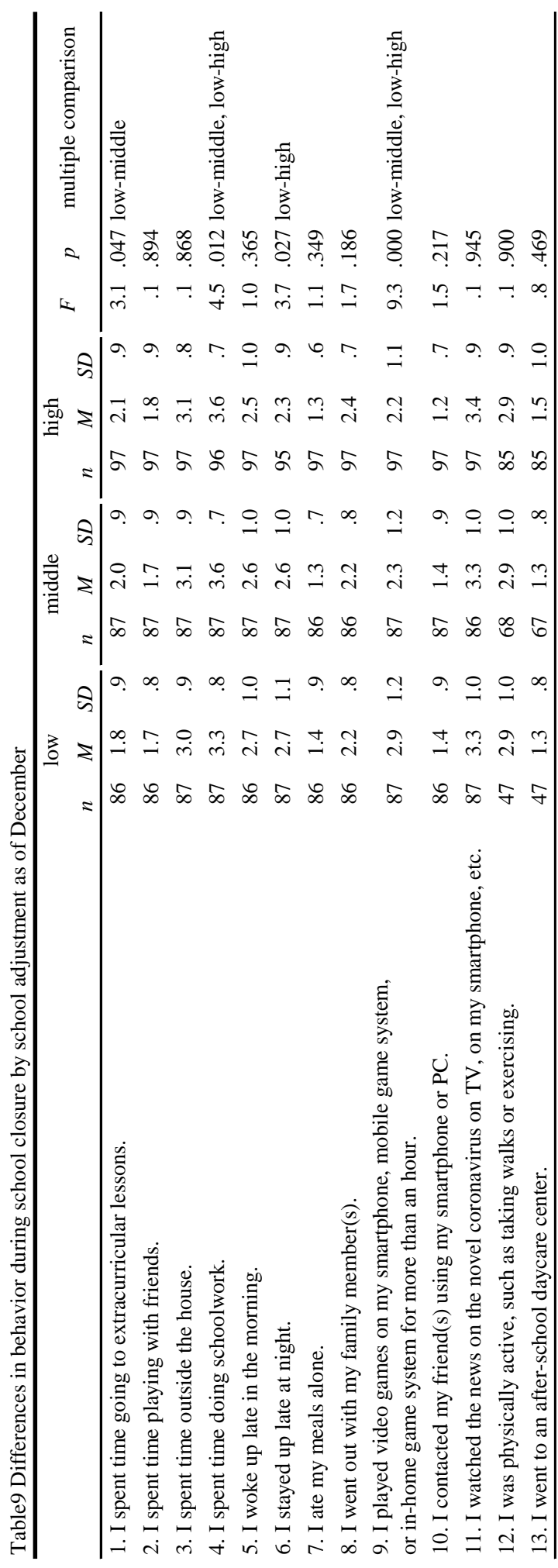

\section{Discussion Overview of Findings}

We investigated the behaviors of schoolchildren during the country-wide school closure and how these behaviors affect children's mental health. We also compared the current and former emotional symptoms using the data obtained before the school closures and examined how the school adjustment before the school closures related to the changes in emotional symptoms.

The analysis using data from the single point in time after school closure indicated that anxiety about daily life after school resumed was associated with emotional problems. Anxiety about catching the disease as well as falling behind in studies was slightly related to emotional problems. At the time of the data collection, there were no confirmed cases of infection in the study area, which could account for the small association between anxiety about infection and emotional problems. However, children who felt more anxious about whether they could return to their normal lives caused more robust emotional problems because of the sudden and unprecedented closure of the school for an unparalleled reason. There was little association between children's emotional difficulties and their behaviors, such as spending time alone during the shutdown.

In an analysis of comparison with the pre-survey data, there was no overall worsening of psychological problems. Although previous studies in other countries had shown that quarantine and social isolation worsened mental health, the

current country-wide school closures in Japan did not lead to worsening of mental health because substantial restrictions were not imposed and the risk of contamination to children was considered relatively low. The fact of no infected cases in the area during the survey does not seem to have contributed to the deterioration of mental health. Rather, the fact that the number of suicides in Japan in April this year was 20\% lower than in the previous year (MHLW, 2020b), and that the reasons for suicides among young people 
were mainly attributed to school (MHLW, 2019), can account for a possible, desirable effect of the school closing on child mental health.

School adjustment as of December did not directly affect the extent of change in emotional symptoms, nor did gender or grade level affect the extent of change. On the other hand, an analysis divided into three groups (low, middle, and high) by scores in December showed no considerable changes in the low and high groups, while the middle group showed a significant worsening of emotional symptoms. Although those who were originally not well school-adjusted had higher emotional symptoms, the school closure likely did not affect their emotional symptoms. The results of the analysis of variance showed that there was no difference between the medium and high groups regarding their behavior during the school closure. Therefore, it is not possible to explain why only the middle group has worsened from the behavior during the school closure. This finding will need to be discussed in more detail in the future.

\section{Need for Support}

The results of this study indicate that at least a one-month-long country-wide school closure does not in itself have a uniformly negative impact on children's mental health. However, it is necessary to provide adequate explanations of their prospects before and during the school closure as the students' anxiety about whether they would be able to return to their daily lives after school resumes, could worsen their mental health. It has also been shown that staying up late led to poor mental health, so support to prevent disruptions in life during school closure would be helpful.

Although school adjustment did not directly explain the worsening of mental health in the analysis involving the levels of school adjustment assessed before school closure, the mental health of the middle group was shown to be deteriorating; therefore, support for children in the middle degree, which is often overlooked, may need to be provided. Furthermore, while this research did not verify impacts connected to the length of the school closure, prior research has indicated that psychological health worsens as the length of quarantine periods increases (Hawryluck et al., 2004). Hence, it seems necessary to keep the length of school closures as short as possible.

\section{Limitations of this Study and Future Issues to Address}

Since this research surveyed schools in a local region where no infections have reported, impacts on children's psychological health could differ in such areas as those where multiple infections have arisen. However, since this research covered a region where anxiety over infection is not significant, it is estimated that this research successfully verified the impact on the psychological health during the school closure period on its own, leaving out the impact of anxiety over contagion on the mental health. Also, it has been shown that infection with COVID-19 tends not to become severe in children, so in that sense, children's anxiety over infection would not have been high. Presumably, a different reaction would be shown if, in the future, an infectious disease severely and widely affects children. Moreover, it has been shown that experiences of quarantine depend on lifestyle and circumstances-specifically, a detrimental impact on psychological health is predicted higher when household income is lower (Cava et al., 2005). This research, however, did not consider household circumstances, so it entailed limitations in that regard. 
As stated above, it is predicted that the psychological impacts of school closures on children will differ significantly according to the presence or absence of alternative measures, the number of infectious patients in proximity, the existence of "stay at home" orders, and others. It is reasonable to argue that there are limitations to generalizing the findings of this research. Further research needs to obtain a more detailed understanding of the psychological impacts that school closures expose schoolchildren.

\section{References}

Braunack-Mayer, A., Tooher, R., Collins, J. E., Street, J. M., \& Marshall, H. (2013). Understanding the school community's response to school closures during the H1N1 2009 influenza pandemic. BMC public health, 13(1), 344. https://doi.org/10.1186/1471-2458-13-344

Brooks, S. K., Webster, R. K., Smith, L. E., Woodland, L., Wessely, S., Greenberg, N., \& Rubin, G. J. (2020). The psychological impact of quarantine and how to reduce it: rapid review of the evidence. Lancet (London, England), 395(10227), 912-920. https://doi.org/10.1016/S0140-6736(20)30460-8

Brymer, M., Layne, C., Jacobs, A., Pynoos, R., Ruzek, J., Steinberg, A., ... \& Watson, P. (2011). Psychological first aid field operations guide. (Hyogo Institute for Traumatic Stress, Trans.) Tokyo: Igaku Shoin. (Original work published 2006)

Cava, M. A., Fay, K. E., Beanlands, H. J., McCay, E. A., \& Wignall, R. (2005). The experience of quarantine for individuals affected by SARS in Toronto. Public health nursing (Boston, Mass.), 22(5), 398-406. https://doi.org/10.1111/j.07371209.2005.220504.X

Furuichi, Y., \& Tamaki, H. (1994). Gakko Seikatsu No Tanoshisa To Sono Kiteiyouin [Factors contributing to the enjoyment of junior high school life]. Bulletin of the Faculty of Education, Okayama University, 96, 105-113.

Goodman R. (1997). The Strengths and Difficulties Questionnaire: a research note. Journal of child psychology and psychiatry, and allied disciplines, 38(5), 581-586. https://doi.org/10.1111/j.1469-7610.1997.tb01545.x

Hawryluck, L., Gold, W. L., Robinson, S., Pogorski, S., Galea, S., \& Styra, R. (2004). SARS control and psychological effects of quarantine, Toronto, Canada. Emerging infectious diseases, 10(7), 1206-1212. https://doi.org/10.3201/eid1007.030703

Jeong, H., Yim, H. W., Song, Y. J., Ki, M., Min, J. A., Cho, J., \& Chae, J. H. (2016). Mental health status of people isolated due to Middle East Respiratory Syndrome. Epidemiology and health, 38, e2016048. https://doi.org/10.4178/epih.e2016048

McArdle, J. J., \& Nesselroade, J. R. (1994). Using multivariate data to structure developmental change. In S. H. Cohen \& H. W. Reese (Eds.), The West Virginia University conferences on life-span developmental psychology. Life-span developmental psychology: Methodological contributions (p. 223-267). Lawrence Erlbaum Associates, Inc.

Ministry of Education, Culture, Sports, Science and Technology. (2020a). Shingata Korona Uirusu Kansensho Taisaku No Tameno Sho, Chu, Kotogakko Ni Okeru Rinji Kyugyo No Jokyo Ni Tsuite [Status of Temporary Closure of Elementary, Junior High and Senior High Schools for the Prevention of Novel Coronavirus Infections]. Retrieved from https://www.mext.go.jp/content/20200304mxt_kouhou02-000004520_1.pdf Accessed 11 March 2020. 
Ministry of Education, Culture, Sports, Science and Technology. (2020b). Shingata Korona Uirusu Kansensho Taisaku No Tameno Shogakkou, Chugakko, Kotogakko Oyobi Tokubetsu Shien Gakko To Ni Okeru Issei Rinji Kyugyo Ni Tsuite (Tsuchi) [About Temporary Closure of Elementary Schools, Junior High Schools, Senior High Schools and Special Needs Schools for the Prevention of Novel Coronavirus Infection (Notice)]. Retrieved from https://www.mext.go.jp/content/202002228mxt_kouhou01-000004520_1.pdf Accessed 23 March 2020.

Ministry of Education, Culture, Sports, Science and Technology. (2020c). Shingata Korona Uirusu Kansensho Taisaku No Tameno Shogakkou, Chugakko, Kotogakko Oyobi Tokubetsu Shien Gakko To Ni Okeru Issei Rinji Kyugyo Ni Kansuru Q\&A No Sofu Ni Tsuite (3 Gatsu 9 Nichi Jiten) [Q \& A on Temporary Closure of Elementary Schools, Junior High Schools, Senior High Schools and Special Needs Schools for the Prevention of Novel Coronavirus Infection (as of March 9)]. Retrieved from https://www.mext.go.jp/content/202000309-mxt_kouhou01000004520_4.pdf Accessed 22 March 2020.

Ministry of Health, Labour and Welfare. (2019). 2019 White paper on Suicide Prevention in Japan. Tokyo, Japan: Nikkei Printing.

Ministry of Health, Labour and Welfare. (2020a). Ibento No Kaisai Ni Kansuru Kokumin No Minasama Eno Message [A message to the public about the event]. Retrieved from https://www.mhlw.go.jp/stf/seisakunitsuite/ newpage_00002.html Accessed 23 March 2020.

Ministry of Health, Labour and Welfare. (2020b). Keisatsucho No Jisatsu Tokei Ni Motozuku Jisatsushasu No Suii Tou [Changes in the number of suicides based on suicide statistics from the National Police Agency]. Retrieved from https://www.mhlw.go.jp/content/202004-sokuhou.pdf Accessed 25 May 2020.

Mizumoto, K., Yamamoto, T., \& Nishiura, H. (2013). Contact behaviour of children and parental employment behaviour during school closures against the pandemic influenza A (H1N1-2009) in Japan. Journal of International Medical Research, 716-724. https://doi.org/10.1177/0300060513478061

Murray, C. J. S. (2010). A collaborative approach to meeting the psychosocial needs of children during an influenza pandemic. Journal for Specialists in Pediatric Nursing, 15(2), 135-143. https://doi.org/10.1111/j.1744-6155.2009.00229.x

NPO Florence. (2020). Issei Kyuko, Kyuen Niyoru Kosodate Setai Eno Eikyo Chosa Kekka Happyo [Announcement of the results of a survey on the impact on childrearing households due to the nationwide closure of schools and preschools]. Retrieved from https://florence.or.jp/news/2020/03/post38598/ Accessed 25 May 2020.

Noda, W., Ito, H., Fujita, C., Nakajima, S., Seno, Y., Okada, R., ... \& Tsujii, M. (2012). Re-examination of the Japanese Version of the Strengths and Difficulties Questionnaire, Parent Ratings Form: Generating cut-off score and normative data for each grade and sex using the entire cohort data in one suburban city in Japan. Clinical Psychiatry, 54(4), 383-391. https://doi.org/10.11477/mf.1405102152

Ozaki, S. (2020, March 11). Ikinuki Mo Dame? Kyukochu No Sotoasobi Ni Tsumetai Shisen Kinjo No Koe Ni Tomadou Hogosha [Can't I take a break? A cold stare is cast on the outside play during the school closure. Parents who are bewildered by the voices of their neighbors]. Mainichi Shimbun. Retrieved from https://mainichi.jp/articles/20200311/k00/00m/040/085000c Accessed 22 March 2020. 
Rubin, G. J., \& Wessely, S. (2020). The psychological effects of quarantining a city. BMJ (Clinical research ed.), 368, m313. https://doi.org/10.1136/bmj.m313

Ryota, G. (2020, March 14). Kyuko Encho Do Sugosu [How will they spend their time with the extended school closure?]. Asahi Shimbun, p.23

Sprang, G., \& Silman, M. (2013). Posttraumatic stress disorder in parents and youth after health-related disasters. Disaster medicine and public health preparedness, 7(1), 105-110. https://doi.org/10.1017/dmp.2013.22

Takahashi, Y. (2017). Personality No Henka To Kenko No Henka No Kankeisei No Kento Wo Okonau: Senzai Henka Model Wo Mochiita 2-Jiten No Judan Data No Bunseki [Examining the relationship between changes in personality and changes in health: Analysis of longitudinal data at two time points using a latent change model]. In K. Shojima (Ed.), Keiryo Personality Shinrigaku [Quantitative personality psychology] (pp.207-218). Kyoto, Japan: Nakanishiya.

The Asahi Shimbun Company. (2009, May 18, evening). Shingata Infuru Kanasen 130nin [The Number of People Infected with the New Flu Has Risen to 130]. Asahi Shimbun, p.1

The Asahi Shimbun Company. (2020, February 28). Zenkoku Shochuko No Kyuko Yousei [Call to Close Elementary, Junior High and Senior High Schools across the Country]. Asahi Shimbun, p.1

Wang, G., Zhang, Y., Zhao, J., Zhang, J., \& Jiang, F. (2020). Mitigate the effects of home confinement on children during the COVID-19 outbreak. Lancet (London, England), 395(10228), 945-947. https://doi.org/10.1016/S0140-6736(20)30547-X 